\title{
基于闪存的可编程线性忆阻器及其存算一体化电路 与系统
}

赵亮 ${ }^{1,3}$ ，高世凡 ${ }^{1}$ ，张圣波 ${ }^{2}$ ，邱翔 2 ，杨帆 ${ }^{1}$ ，李杰 ${ }^{3}$, 陈泽志 $^{3}$ ，赵毅 $1^{*}$

1. 浙江大学信息与电子工程学院, 杭州 310027

2. 上海闪易半导体有限公司, 上海 201210

3. 合肥睿科微电子有限公司, 合肥 230088

* 通信作者. E-mail: yizhao@zju.edu.cn

收稿日期: 2021-05-10; 修回日期: 2021-07-27; 接受日期: 2021-08-09; 网络出版日期: 2022-01-06

科技创新 2030 - “新一代人工智能” 重大项目 (批准号: 2020AAA0109001)、浙江省重点研发计划 (批准号: 2021C01039) 和中央 高校基本科研业务费专项资金 (批准号: 2020XZZX005-06) 资助项目

摘要＼cjkstart基于新型非易失性存储器和存内计算架构的神经网络推理芯片在功耗、速度和存储密度等方 面展现出突出的优势, 使其在物联网和边缘计算等应用领域受到广泛关注。在本文中, 我们详细介绍 了一款基于可编程线性忆阻器 (programmable linear random-access memory, PLRAM) 的存算一体化 片上系统芯片的设计和实现方法. 为了在资源受限条件下实现高效的推理计算, 该系统结合了器件、 电路和系统层面的一系列新技术, 包括具备每单元 7 比特存储能力的新器件、数据自适应写入、电路 失配补偿, 以及基于残差模型训练的模型部署技术。这些新技术帮助该系统实现优越的整体性能, 使 其具有紧凑的外观尺寸, 超过 $10 \mathrm{TOPS} / \mathrm{W}$ 的能效和接近 $95 \%$ 的计算精度, 并被成功应用于一款低功 耗、低成本的语音多关键词识别产品.

关键词存算一体化, 片上系统, 改进型浮栅晶体管, 可编程线性忆阻器, 模型部署技术

\section{1 引言}

近年来, 融合了人工智能算法的感知计算系统被逐渐应用于物联网领域, 形成了一类新型的技术 生态 —— 智能物联网 (artificial intelligence of things, AIoT). 智能物联网硬件的特点是将计算能力尽 量向边缘终端移动, 并在此基础上充分运用人工智能算法, 加速端侧数据处理和交互感知的能力. 具 体而言, 端侧人工智能算力能够对传感器所产生的海量数据 (如图像、语音等) 进行预处理, 提取出有 效特征后再进行传输或本地消化使用, 从而缩减单个传感器所需的网络带宽. 在带宽一定的前提下, 智能物联网终端允许部署数量和种类更多的传感器, 从而为物联网设备带来更丰富的功能和更好的用

\footnotetext{
引用格式: 赵亮, 高世凡, 张圣波, 等. 基于闪存的可编程线性忆阻器及其存算一体化电路与系统. 中国科学: 信息科学, 2022, 52 : 176-186, doi: 10.1360/SSI-2021-0157

Zhao L, Gao S F, Zhang S B, et al. Programmable linear random-access memory and its in-memory computing circuits and systems based on flash memory (in Chinese). Sci Sin Inform, 2022, 52: 176-186, doi: 10.1360/SSI-2021-0157
} 
户体验. 目前, 在个人可穿戴设备、智能家居、智慧城市和工业自动化等领域中, 智能物联网的应用模 式正得到广泛的研究和探索, 以期实现更好的服务质量 (quality of service, QoS)、人机交互和实时决 策效果. 伴随着这一发展趋势, 电路和系统设计者也面临着新的技术挑战, 包括但不限于:

- 随着传感器技术的发展, 能通过物联网设备收集和处理的数据量呈现爆炸性增长;

- 端侧的通信带宽有限, 即使采用 $5 \mathrm{G}$ 技术也难以跟上数据量增长的步伐;

- 端侧的能源供应有限, 对边缘侧人工智能算力的能效提出了更高的要求;

- 传统的硬件架构在处理数据密集型的人工智能任务时功耗较高、效率较低.

为了应对这些挑战, 新的计算范式和系统设计方法 (如存内计算、近存计算等) 正受到越来越多的 关注. 以存内计算为例, 其主要动机是解决传统冯 ·诺依曼 (von Neumann) 架构的计算系统中 “存储 墙” 的问题. 这类问题的根源是: 在传统冯. 诺依曼架构中计算和存储单元是分离的, 因此在处理深度 神经网络 (deep neural network, DNN) 之类的数据密集型任务时, 需要在处理器和存储器之间频繁地 交换数据, 从而形成了系统性能和功耗的一个瓶颈 ${ }^{11]}$. 相比之下, 存内计算范式将计算和存储功能整 合到同一个存算一体化单元之中, 从而克服了存储墙问题, 在单位能效、成本和计算速度方面提供了一 些显著优势 ${ }^{[2]}$. 根据所采用的存储器技术的不同, 存算一体化单元又可以分为易失性和非易失性两类. 前者通常基于 SRAM (static random access memory) 或者 DRAM (dynamic random access memory), 需要在系统启动时将计算模型重新加载到存储器阵列当中, 后者 (包括 RRAM (resistive random access memory), PCM (phase change memory), MRAM (magnetic random access memory) 等新型存储器技术 在内) 则没有此类限制. 由于非易失性存算一体化单元能消除静态功耗, 并使得系统在恢复供电时能 够即时开启 (无需重新加载模型和算法), 因此日益成为智能物联网应用的首选技术之一 ${ }^{[3]}$.

本文介绍了一款基于存算一体化技术的语音识别片上系统 (system on chip, SoC) 芯片的设计和 实现细节, 该系统采用改进型浮栅晶体管器件做为神经网络加速器, 并结合了制造工艺、电路设计和 系统集成方面的一系列创新技术和技巧, 其目标应用是在终端资源 (功耗、带宽等) 受限情况下的语音 关键词识别. 本文的主要创新点归纳如下:

- 基于改进型浮栅晶体管器件和微秒级自适应写入技术, 实现了每单元 7 比特的存储功能, 从而 最大程度地提高权重数据的存储密度并降低芯片测试成本.

- 提出了基于迁移学习思想的模型部署技术, 采用训练残差模型的方法对部署后的模型参数进行 微调, 从而显著改善了权重部署过程中的精度退化问题.

- 基于存算一体化 $\mathrm{SoC}$ 实现了一款语音多关键词识别产品, 具有体积小、功耗低和价格低等优势, 在实时处理中能实现大于 $10 \mathrm{TOPS} / \mathrm{W}$ 的能效和 $94.8 \%$ 的计算精度.

\section{2 基于改进型浮栅晶体管的存算一体化片上系统}

\section{1 改进型浮栅晶体管}

本文的存算一体化片上系统的核心是由改进型浮栅晶体管构成的存算一体化阵列, 用于加速神经 网络中向量和矩阵相乘这一关键操作. 实际应用中, 通常采用字线上的输入电压来表示输入向量的值, 采用阵列中每个交叉点的器件电导来表示神经网络模型的权重, 通过测量位线上累积的电流之和得到 乘加运算在每个维度上的结果. 在这样的计算范式中, 根据基尔霍夫 (Kirchhoff) 电压和电流定律可知, 如果要进行多比特权重的运算, 则器件电导应和权重值呈严格的线性关系.

本文中, 电导的连续调节是通过控制改进型浮栅晶体管的阈值电压而实现的. 虽然以 ETOX 结 

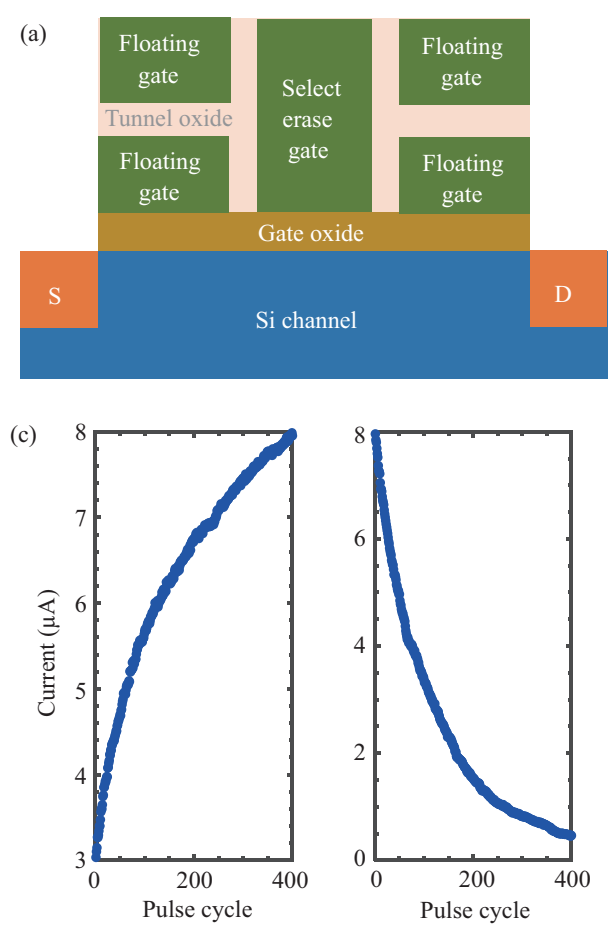

(b)
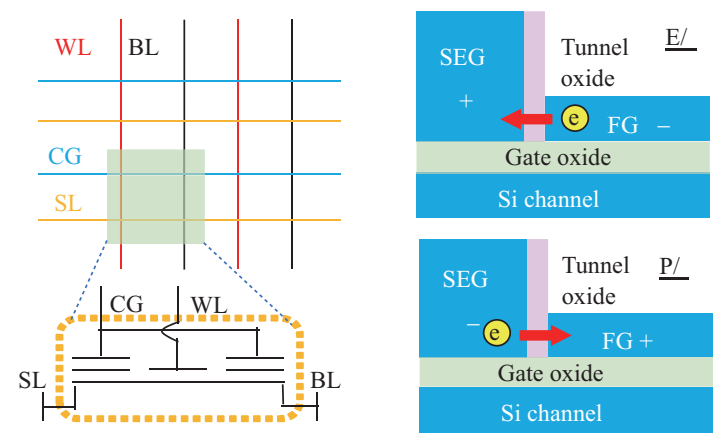

(d)

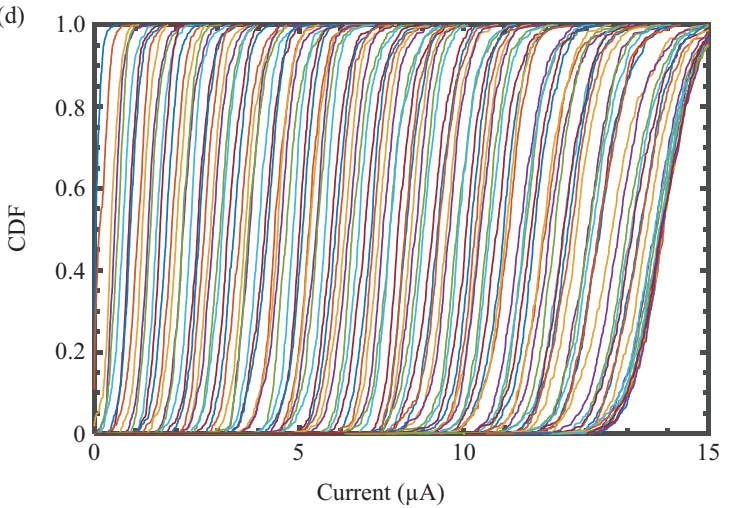

图 1 (网络版彩图) (a) PLRAM 器件结构示意图; (b) 对器件进行逐步擦除和编程操作的示意图; (c) 单个 PLRAM 器件对固定擦除和编程电压的响应; (d) 在 $876 \times 128$ 阵列分块上的编程结果, 显示阵列具有 7 比特/单 元的编程能力

Figure 1 (Color online) (a) Illustration of PLRAM devices ${ }^{[4]}$; (b) operation scheme for gradual erasing and programming; (c) single cell current response of PLRAM to constant-voltage erase pulses; (d) programming results of an $876 \times 128$ sector, demonstrating 7-bit/cell capability

构 $[4,5]$ 为代表的浮栅晶体管器件长期以来都被作为多态存储器使用, 但是存算一体化应用对于多态存 储的要求和传统存储器存在差异: 传统存储器读数据时往往采用恒定电压, 而神经网络计算中, 电压 由输入信号决定而非恒定, 因此要求器件在整个工作区间内都处于线性区, 可以使用的阈值电压范围 相比存储应用也更为有限. 为了解决这一问题, 团队在前期工作中提出了一种改进型的浮栅晶体管器 件 ${ }^{[6]}$, 并将其命名为可编程线性随机存储器 (programmable linear random-access memory, PLRAM). 该器件的结构特点是具有两组浮栅 (floating gate, FG) 和控制栅 (control gate, CG) 堆叠在一起形成 的 Etox 结构, 两组结构之间则有一个选择擦除栅 (select erase gate, SEG) 用于选通和横向擦除操作. 通过使用双向 F-N 隧穿, 这一新型器件实现了浮栅中电子高度可控的注入 (编程) 和移除 (擦除) 调 节 (图 1(a) (c)). 在注入电子时, SEG 端将被抬升至高电压, 而 CG 端将被拉至低电压, 从而实现基于 F-N 隧穿的电子注入 (图 1(b)). 这一操作模式不同于传统闪存所使用的基热载流子注入, 能够更高效 且准确地控制电子数的变化. 进一步地, 通过自适应的编程和擦除迭代, PLRAM 阵列能够在兆字节规 模上实现 7 比特/单元的编程精度 (图 1(c) 和 (d)), 从而保证器件电导和权重值的精确对应. 结合并行 输入和输出, PLRAM 能够作为神经网络加速器中的基本突触单元实现高效的存内计算.

\section{2 系统架构}

图 2(a) 展示了基于改进型浮㮽晶体管所设计的存内计算 $\mathrm{SoC}$ 的系统架构. 该系统包括 PLRAM 器 


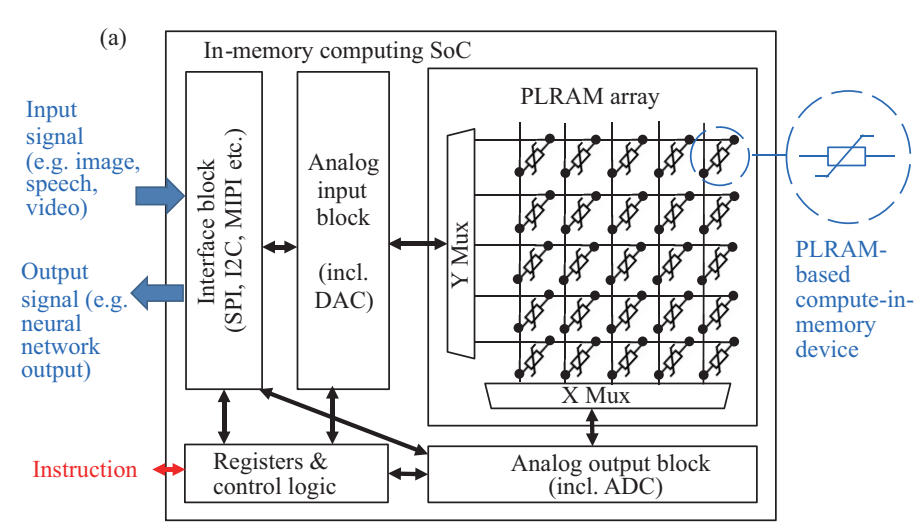

(b)

Die photo of CIM SoC

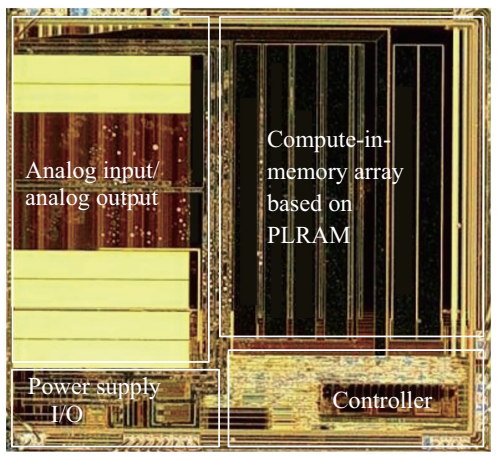

图 2 (网络版彩图) (a) 基于改进型浮栅晶体管设计的存算一体化片上系统的芯片架构, 包含了存算一体化阵列、模 拟输入和输出模块、控制逻辑和接口模块; (b) 存算一体化片上系统的实物照片 (Hexagonal 芯片)

Figure 2 (Color online) (a) System architecture of the in-memory computing SoC designed based on novel flash memory; (b) die photo of the in-memory computing SoC chip (Hexagonal-A01) based on novel flash memory

件组成的存算一体化阵列、模拟输入模块 (包含了数模转换单元 DAC (digital-to-analogue conversion))、 模拟输出模块 (包括模数转换单元 ADC (analogue-to-digital conversion))、控制逻辑模块和 I/O 接口 模块. 按照前文所述的计算范式, 语音或视频流等输入信号在进入存算一体化阵列前, 首先将通过接 口模块, 然后由模拟输入模块转化为模拟信号并作用于阵列的字线. 运算完成后, 模拟输出模块会检 测位线上的电流值并将其转化为计算结果, 再经过 $\mathrm{I} / \mathrm{O}$ 接口输出结果.

本文中的存算一体化 SoC 芯片是基于 $90 \mathrm{~nm}$ CMOS (complementary metal oxide semiconductor) 工艺平台实现的 (图 2(b)), 该工艺平台提供的嵌入式闪存解决方案能够实现 PLRAM 存算一体化单 元所需的工艺流程. 本文中单个 SoC 芯片的存储阵列规模达到 $2048 \times 3096$, 合计有超过 600 万个新型 PLRAM 单元. 假设每单元能够存储 7 比特权重数据, 则单芯片能实现的权重数据存储容量为 42 兆 位, 广泛适用于语音识别应用中的一些常用神经网络模型. 该存储阵列的工作模式分为数据模式和存 算模式. 在数据模式中, 该阵列的所有存储单元可采用统一的方法寻址和写入、读取操作, 类似于常见 NOR Flash 的操作模式; 在存算模式中, 可以通过行列选择器选通阵列中的一个子阵列进行乘加计算. 在处理多层神经网络的计算时, 每层的输出会暂存到容量为 $10 \mathrm{~Kb}$ 的 SRAM 缓存中并用于下一层输 入, 直至最终向片外输出控制信号. 在 2.3 和 2.4 小节中, 将详细讨论模拟输入和模拟输出模块的详细 设计方案.

\section{3 模拟输入模块的设计}

图 3 展示了包含输入缓冲器和 8 位数模转换功能的模拟输入模块的设计方案. 在这一设计中, 精 度为 8 位的输入数据被按位分开, 以高低电平的形式同时施加到 8 个上拉晶体管的栅极. 每个上拉晶 体管的沟道宽长比 $(W / L)$ 按照 1:2:4:8:16:32:64:128 的比例设计以对应不同的位权, 它们的作用是能将 输入的高低电平转换为等比例的电流并汇聚到输出端.

如图 3(a) 所示, 由输入数据产生的上拉电流可以通过一个电阻转化为输出电压, 并施加到存算一 体化阵列的输入缓冲器上. 但是, 这样的设计存在一定缺陷: 每条输入数据的字线都需要使用一组数模 转换器和缓冲器, 因此不同输入字线之间电阻阻值和晶体管特性可能会产生较大的波动, 造成失配问 题从而降低存算一体化的计算精度. 为了补偿输入电路的失配问题, 本文提出了如图 3(b) 所示的模拟 输入模块设计, 该设计采用一个浮㮽晶体管代替电阻来产生输入电压. 采用这样的设计, 可以通过调整 
(a)

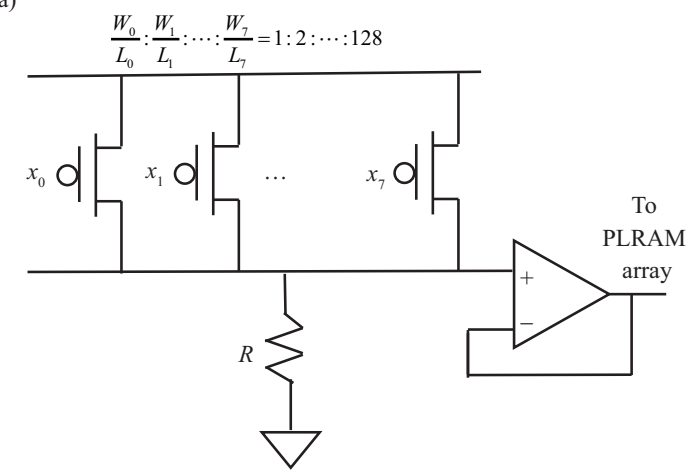

(b)

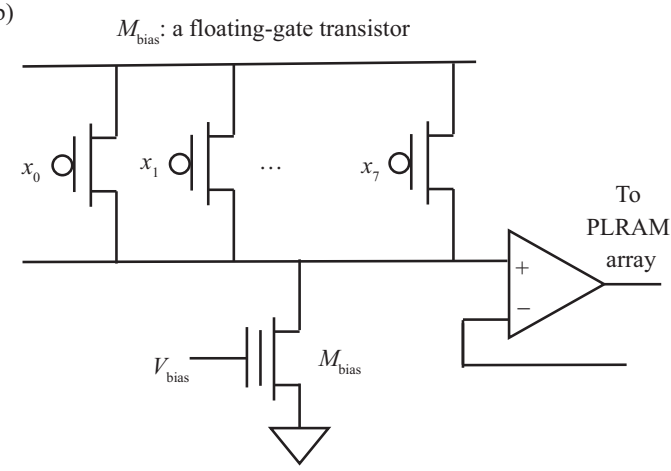

图 3 模拟输入模块的电路原理图. (a) 按位权设计的上拉晶体管用于实现数模转换, 并通过下拉电阻转为输出电压; (b) 采用等效电阻可调节的浮栅晶体管代替下拉电阻, 用于解决电路失配问题

Figure 3 Circuit diagram of the analog input block. (a) DAC based on pull-up transistors and a pull-down resistor; (b) $\mathrm{DAC}$ with a trimmable floating-gate transistor replacing the pull-down resistor, which helps to compensate the mismatch problem of the circuits

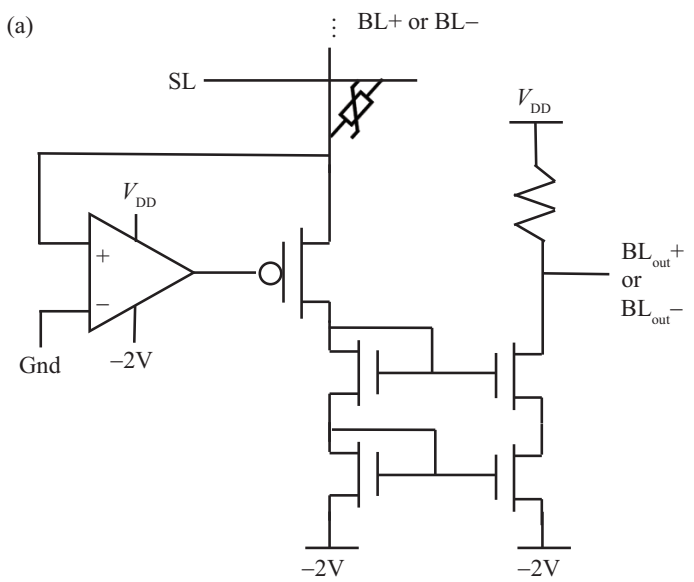

(b)

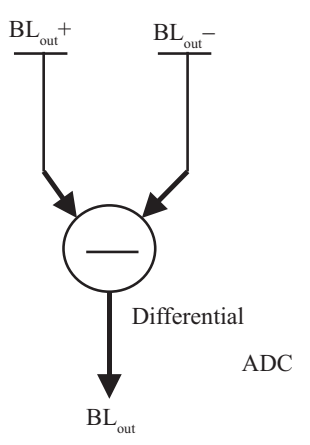

图 4 模拟输出模块的电路原理图. (a) 带有两级级联电流镜的感测放大器, 用于生成输出电流或电压; (b) 差分模 数转换器, 用于将存储正负权重的两条位线的累加结果相减并输出计算结果

Figure 4 Circuit diagrams of the analog output block. (a) Sense amplifier with cascade current mirror for output voltage sensing; (b) schematics of positive and negative output subtraction with differential ADCs

阈值电压来改变浮栅晶体管 (Mbias) 的等效电阻, 使得所有数模转换器针对相同的输入数据产生一致 的输出模拟电压, 从而消除彼间的失配. 这一配置阈值电压的过程又被称为电路参数匹配 (trimming).

\section{4 模拟输出模块的设计}

图 4 展示了模拟输出模块的设计方案. 该模块由一组能够精确复制电流的两级级联电流镜和一个 用于检测输出结果的差分模数转换器组成. 级联电流镜可以将位线上各存算一体化单元累加的电流 精确复制到旁路上, 用于产生输出电压并隔绝干扰. 本文中的存算阵列采用了正负权重分离存储的方 案, 即相邻的一对位线上分别存储计算模型中权重的正值分量和负值分量, 而差分模数转换器的作用 则是将两条位线的电流和相减并输出计算结果. 该模数转换器的输出精度为 $10 \mathrm{bit}$. 在存算一体化单 元存储精度不变的前提下, 这样的设计有效地扩展了权重值的动态范围, 等效于增强了神经网络的计 
(a)

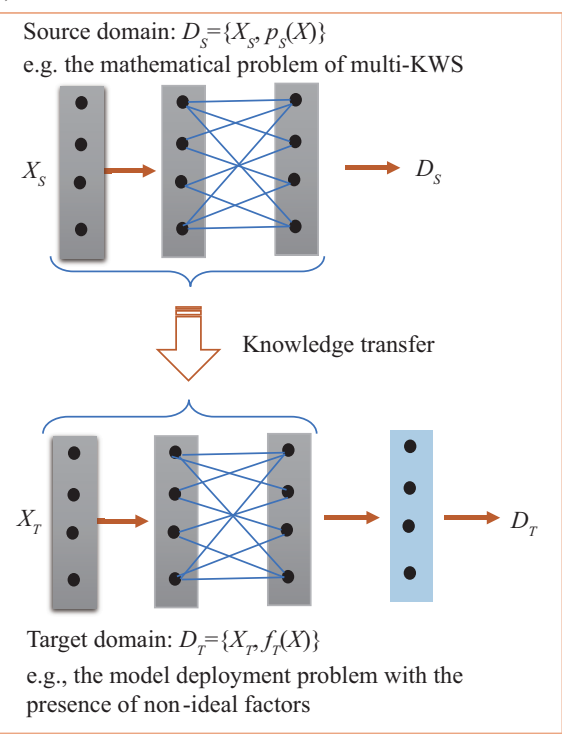

(b)

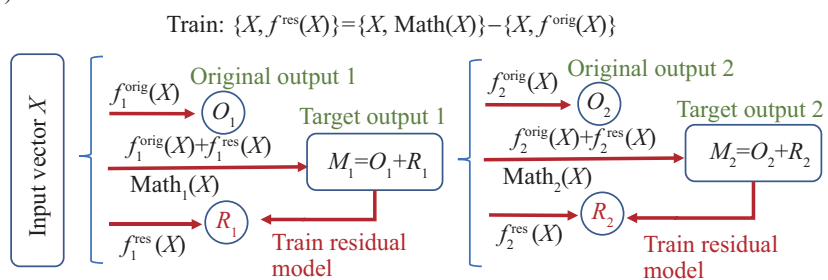

(c)

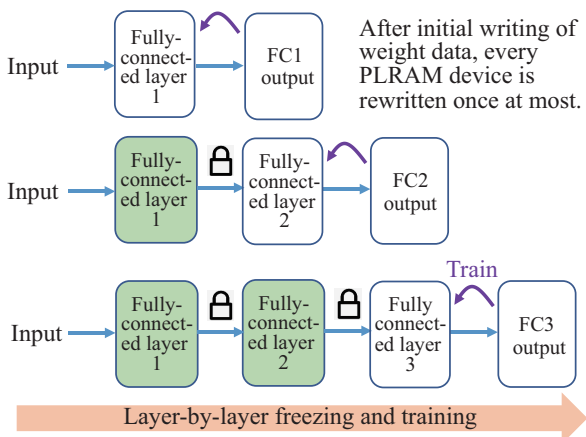

图 5 (网络版彩图) 器件非线性和 IR 压降问题. (a) 器件的非线性引起不可忽视的权重误差; (b) IR 压降模拟计 算: 在集成阵列 $(30 \times 30)$ 中的字线、位线互联线上出现明显的压降

Figure 5 (Color online) Issues of device nonlinearity and IR drop. (a) Device nonlinearity causes inevitable weight error with significant impact; (b) simulation of the IR drop effect: severe voltage drop is observed in the BLs and WLs of a $30 \times 30$ array

算准确度 ${ }^{[7]}$. 此外, 通过对两条位线上的电流进行相减, 可以消除检测电路所产生的一些系统误差 (如 阈值电压漂移等), 从而得到更准确的计算结果.

\section{3 神经网络模型在存算一体化芯片中的部署方法}

\section{1 采用存算一体化芯片计算神经网络时的误差溯源}

基于神经网络的推理计算对于智能物联网具有重要价值, 其核心诉求是在资源有限的情况下实现 较高的推理准确率. 为了实现这一目标, 就需要全面了解存算一体化范式及其系统中所存在的非理想 因素, 并通过分析各个非理想因素对推理误差的贡献, 有针对性地消除主要误差来源. 这些非理想因

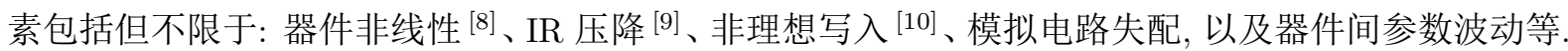

如图 5(a) 所示, 器件非线性指的是存储单元的电阻随输入电压变化的趋势不符合线性关系. 在向 量和矩阵的相乘运算中, 这一特性造成的结果是存算一体化单元所存储的等效权重值会随着输入电压 的变化而改变, 从而大大降低了其计算准确性. IR 压降, 又称为电阻压降, 指的是集成电路互连线在 电流通过时产生的电压下降现象. 随着集成电路特征尺寸的不断缩小, 由互连线电阻引起的电压降现 象也变得日益严重, 图 5(b) 是采用电阻网络方法 [11] 模拟的交叉阵列结构中的导线电压降. 由此可见, 特别是对于大容量存内计算阵列, IR 压降会导致阵列两端存算单元的实际电压产生较大漂移, 从而造 成不可忽略的计算误差. 模拟电路失配问题在前文模拟中已经有所讨论, 其表现形式是各器件、电路 模块之间的参数不一致、不匹配从而造成计算准确率的退化, 其主要成因包括电路设计的仿真结果和 实际成品的参数偏差、芯片制造过程中的随机工艺波动, 以及温度变化等外部因素. 最后, 器件与器件 之间的参数波动在图 1(d) 中有直观体现, 通过自适应编程、权重复制等方法可以较大程度地消除其 
(a)

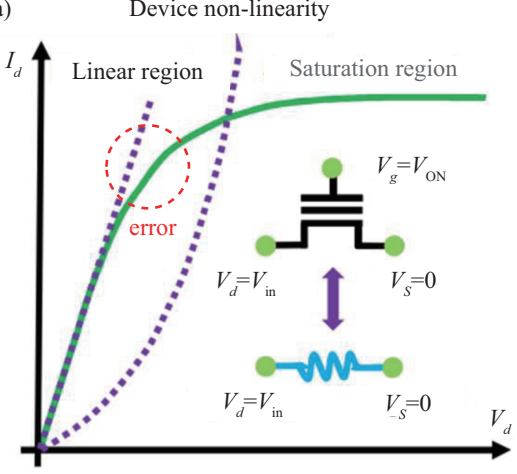

(b) IR drop simulations

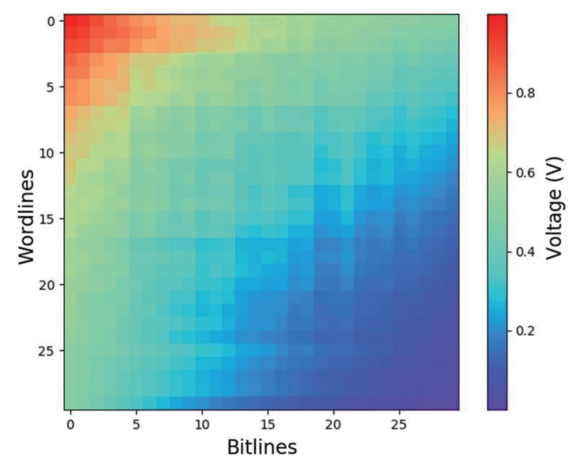

Simulated condition:

$R_{\mathrm{MEM}}=2 \mathrm{k} \Omega$

$R_{\mathrm{wL}}=50 \Omega$

$R_{\mathrm{BL}}=50 \Omega$

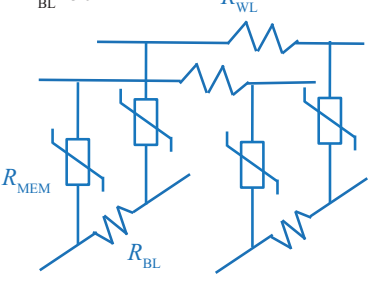

图 6 (网络版彩图) (a) 迁移学习的概念及其在神经网络模型部署问题中的对应; (b) 逐层训练残差模型的方法示意 图, 其中在存算一体化 SoC 芯片中部署原始模型后得到的实际输出为 $O 1$, 采用原始模型和纯数学方法计算得到的 目标输出为 $M 1$, 残差模型的训练目标 $R 1=M 1-O 1$, 使用该方法时每个权重数据最多只需要更新一次; (c) 逐层 冻结 - 残差训练方法, 以保证整体准确率的提升不随神经网络层数增加而衰减.

Figure 6 (Color online) (a) Transfer learning concepts and their applications in model deployment; (b) the method of training a residual model only needs one-time update after the initial deployment; (c) progressive layer-by-layer freezing and training approach

影响. 通过对存内计算的误差来源进行物理分析, 研究人员发展了一系列技术用于减轻和消除特定的 误差来源的影响. 例如, 前文所述的权重差分表示的思路, 通过在两条不通的位线上分别存储正权重和 负权重, 以抵消基于相变存储器 $(\mathrm{PCM})$ 的存算一体化阵列在写入权重时的不对称行为, 并扩展其存 储权重的动态范围 ${ }^{[7]}$. 在另一项工作中, 研究者使用 SPICE 建模和模拟仿真来预测基于阻变存储器 的二进制神经网络加速器中的 IR 压降的幅度并提出了改进方案 ${ }^{[9]}$. 另一方面, 在实际应用中, 存内计 算系统可能包含多方面的非理想因素, 要对这些因素进行独立的分析和解决具有较大的挑战性. 因此, 需要一种系统性的模型部署方法, 该方法能够整体改善各类非理想因素造成的误差, 使得推理运算的 准确率接近于理论值; 同时, 采用这种方法部署时不需要过多的有关计算模型和硬件特性的前置信息, 从而保证模型部署的所需的时间和成本基本不变. 正是基于这样的背景, 本文采用了基于片上迁移学 习的模型部署方法, 在 3.2 小节将作详细讨论.

\section{2 基于采用片上迁移学习的模型部署和微调}

图 6(a) 总结了迁移学习的概念及其在神经网络模型部署到存算一体化阵列中这一问题上的对应 关系. 在迁移学习中, 源域代表已有的知识, 目标域代表要学习的新知识、解决的新问题, 迁移学习的 应用条件通常是源域和目标域的问题在结构上具有相似性. 特别地, 在深度学习领域中, 迁移学习研 究如何将已有神经网络模型应用到和源域不同的、但是有一定关联的新领域中, 有机地利用源域的知 识对目标域进行更准确和更高效地建模. 以存算一体化语音应用为例, 源域可以是基于语音信号的多 关键词识别这个已充分解决的数学问题, 而目标域则是在已知神经网络结构和权重的基础上, 根据存 算一体化阵列特性进行的权重数据优化 (也称为硬件优化权重), 以实现在权重写入后存算一体化芯片 的计算结果尽可能接近源域中纯数学模型预测的结果. 这里的目标域问题涉及到存算一体化阵列中复 杂的非理想因素, 如非线性、IR 压降、器件参数波动和电路失配等, 因此不同于源域问题. 同时, 这两 个域的问题在数学结构上又具有明显的相似性 (相同的网络结构). 根据迁移学习的理论, 这一类问题 常常被称为 “域适应” [12], 可以借鉴 “模型微调”相关的技术加以解决. 
在本文中, 我们借鉴了 $\mathrm{He}$ 等 ${ }^{[13]}$ 提出的深度残差学习技术以解决神经网络模型部署中的模型微 调问题, 这一方法提出的初衷是为了解决深度神经网络在训练过程中的梯度消失问题从而减少训练时 间. 如图 6(b) 所示, 尝试在存算一体化芯片中部署模型参数后, 会观察到非理想因素引起的准确率退 化 (原始输出和目标输出之差). 此时, 我们不直接在芯片上对权重进行训练和优化, 而是首先训练一个 残差模型, 其训练目标是硬件优化权重与原始权重之差. 与传统的片上在线训练方法相比 ${ }^{[14,15]}$, 这种 训练残差模型的方法可以大大减少写入存储单元的次数, 从而为在非易失性存算一体化阵列提供了一

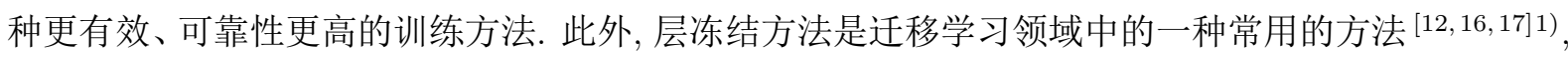
常用于在问题发生变化但模型结构不变的情况下对模型参数进行微调. 采用层冻结方法的主要驱动力 包括：目标域的新问题所允许的训练集规模有限，且新旧问题之间存在类似的低阶特征可以共用 (例 如本文所述的模型部署问题). 层冻结方法的一个普遍的好处是: 采用更小的训练集就能针对新问题达 到满足要求的识别准确率. 在实际操作中, 本文采用了一种逐层冻结 - 残差训练的新方法, 具体的训 练流程如图 6(b) 和 (c) 所示. 首先, 将理论模型 (采用 GPU 等设备进行训练、已达到理想的准确率) 部署到存算一体化阵列中, 并使用训练集通过神经网络第 1 层的输出结果来训练第 1 层的残差模型; 其次, 我们将训练完成的残差模型更新到第 1 层网络的部分, 然后使用训练集连续通过网络前两层得 到的输出来训练第 2 层的残差模型; 逐层重复此训练过程, 直到整个深度神经网络模型都在存算一体 化阵列中得到了训练. 在此过程中, 每个权重数据最多只需要更新一次, 从而最大程度地减少了非易 失性存储器的耐擦写特性所带来的限制. 这一逐层冻结 - 残差训练方法是层冻结的一种特殊形式, 是 结合了存算一体化阵列的特性和语音关键词识别应用需求的一种可操作性较强的方法. 首先, 闪存器 件擦写次数有限且擦写时间远大于读取时间, 决定了对整个网络进行大样本在线训练是不现实的, 而 逐层冻结 - 残差训练的方法对于单层线性权重的训练来说和在线训练是等价的 (不考虑激活函数的 单层感知器等价于多元线性回归). 另一方面, 采用经典反向传播算法训练时, 其反向传播的梯度会随 层数的增加而减小甚至消失 ${ }^{[18]}$, 但硬件非理想因素产生的误差却会在反向传播过程中积累. 通过逐 层冻结 - 残差训练的方法, 可以阻断非理想因素造成的误差反向传播, 同时前序层中某些难以通过训 练消除的误差模式 (如特定工艺偏差等) 尚有可能在后续层的训练过程中得到补偿. 综合考虑这些因 素, 在存算一体化芯片模型部署这一问题上, 逐层冻结 - 残差训练的方法应当是比基于传统反向传播 的在线训练更为有效的方法.

图 7 总结了基于残差模型训练的神经网络部署方法的准确率, 并和部署原始权重或采用基于 IR 压降模型的启发性修正方法时的准确率进行了比较 [9]. 实验采用了一个结构简单的语音识别模型, 包 含了快速傅里叶变换 (fast Fourier transform, FFT)、梅尔系数提取和以梅尔系数为输入的 3 层感知器 (multi-layer perceptron, MLP), 全部采用存算一体化芯片计算. 该模型的权重参数是在一商业化语音 数据集基础上通过训练得到的. 该数据集由 10 个语音关键词以及其他无关的语音片段组成. 其中, 每 个语音片段的时长为 $1.522 \mathrm{~s}$, 采样率为 $8 \mathrm{MHz}$, 语音片段总长度为 12176 , 每个关键词语音都落位对齐 到语音片段的结尾. 数据集的规模为: 每个关键词对应的语音片段约 40000 条, 无关语音片段总数约为 1000000 条. 在实验过程中, 为比较计算准确率, 统一采用测试集在存算一体化芯片上和理论模型中得 到的输出向量之间的相关系数作为准确率的量化标准. 对于每一层网络, 使用了具有 10000 个输入向 量 (长度为 256) 的小型训练集和反向传播算法进行了残差模型的训练. 结果表明, 基于残差模型训练 的部署方法可以极大地提高存算一体化阵列的推理准确率. 而且, 该方法在每一层网络上能产生类似 的准确率提升: 和原始权重相比, 每层提高约 3\%; 和启发式修正方法相比, 每层提高约 $1 \%$; 并未随着 网络深度加深而减小. 这一结果说明受迁移学习启发而提出的逐层冻结和训练残差模型的方法是有效

1) Keras developer guide: transfer learning \& fine tuning. https://keras.io/guides/transfer_learning/. 

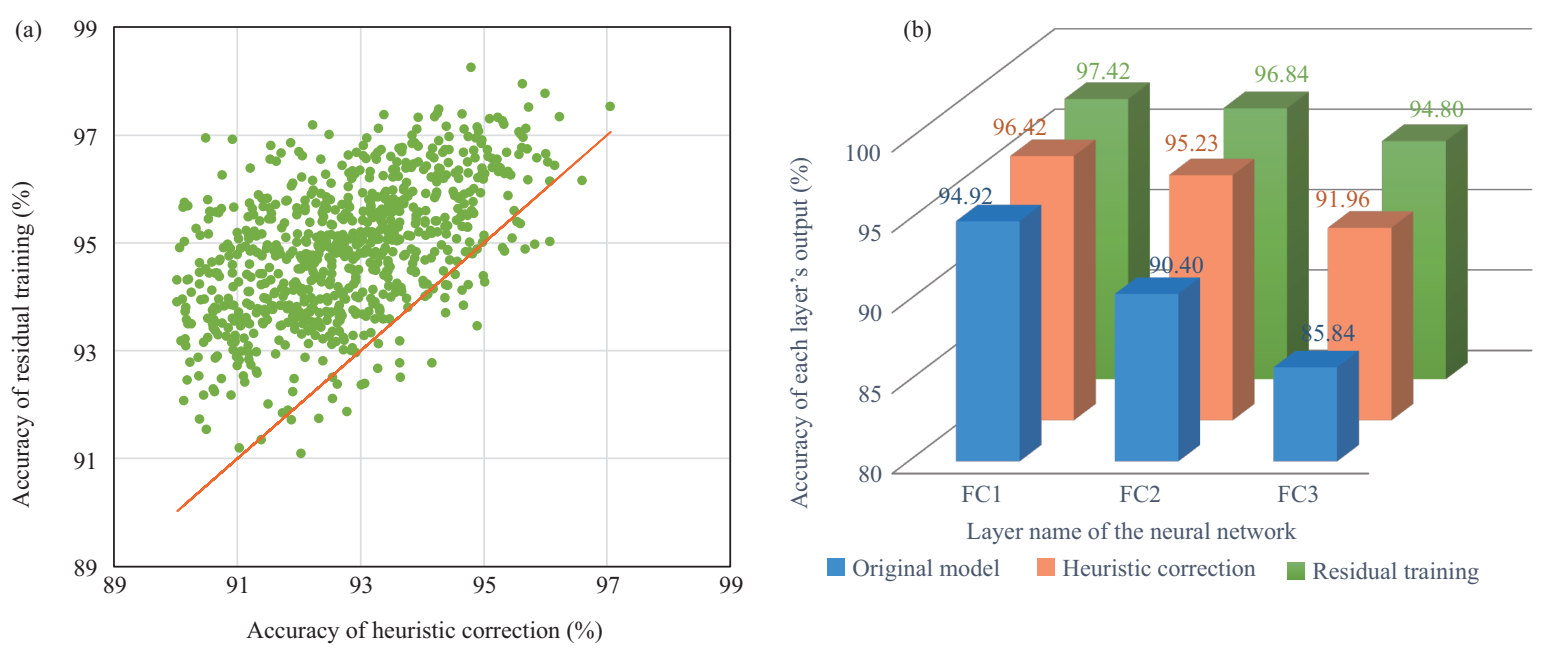

图 7 (网络版彩图) (a) 采用残差模型训练方法和启发性修正方法后, 相同输入向量的计算准确率的比较; (b) 神经 网络各层输出的准确率比较, 包括原始权重、启发性修正方法和残差模型训练方法得到的结果

Figure 7 (Color online) (a) Comparison of post-FC3 accuracy between the residual-training approach and heuristic correction, each point corresponds to one input vector in the test set; (b) post-layer accuracy improvements for each FC layer with the residual-training approach, compared to the original model and the heuristic correction approach

的, 且适用于更深层的神经网络. 整体来看, 上述模型部署方法将语音关键词识别模型的综合推理准 确率从约 $86.0 \%$ 提高到了约 $94.8 \%$, 大大改善了应用于实际产品时的用户体验.

\section{4 语音关键词识别模组的集成和验证}

本文所设计的基于新型浮栅晶体管的存算一体化 $\mathrm{SoC}$ 具备从语音信号处理、特征提取到关键词 识别和结果输出的完整功能, 为相关应用提供了一种紧凑型的解决方案. 图 8(a) 展示了一个具体的系 统集成案例, 该系统中除了存算一体化 SoC 以外无需其他集成电路芯片, 即可执行从 FFT 信号处理 到深度神经网络计算和结果输出的全套功能.

目前, 该系统已经被运用于智能家居产品中, 提供语音多关键词识别的功能. 如图 8(b) 所示, 最终 产品由一个 $3 \mathrm{~V}$ 纽扣电池供电, 在执行连续的语音关键词识别任务时, 通过测试电压和电流可验证其 能效达到 $10 \mathrm{TOPS} / \mathrm{W}$ 以上. 图 8(c) 总结了本文设计的存算一体化 $\mathrm{SoC}$ 在多关键词识别应用中的整 体性能, 并与采用 CPU、GPU、CMOS, 以及神经形态芯片的现有解决方案进行了比较 ${ }^{[19]}$. 从比较结 果来看, 存算一体化 SoC 芯片具有明显的成本优势, 以及领先于现有方案的高能效.

\section{5 结论}

本文设计和实现了一款基于新型浮栅晶体管的存算一体化片上系统芯片, 并演示了其在语音多关 键词识别任务中的应用. 研究过程中, 结合了从器件、电路到系统层面的一系列新技术以实现高效的 神经网络推理和信号处理. 创新性的每单元 7 比特数据存储、自适应写入和电路失配补偿技术帮助该 系统实现了优越的整体性能. 此外, 本文还探索了基于迁移学习概念的神经网络模型部署技术, 通过 逐层递进的残差模型训练方法, 极大地改善了权重数据部署时的准确率退化问题. 最终实现的语音识 

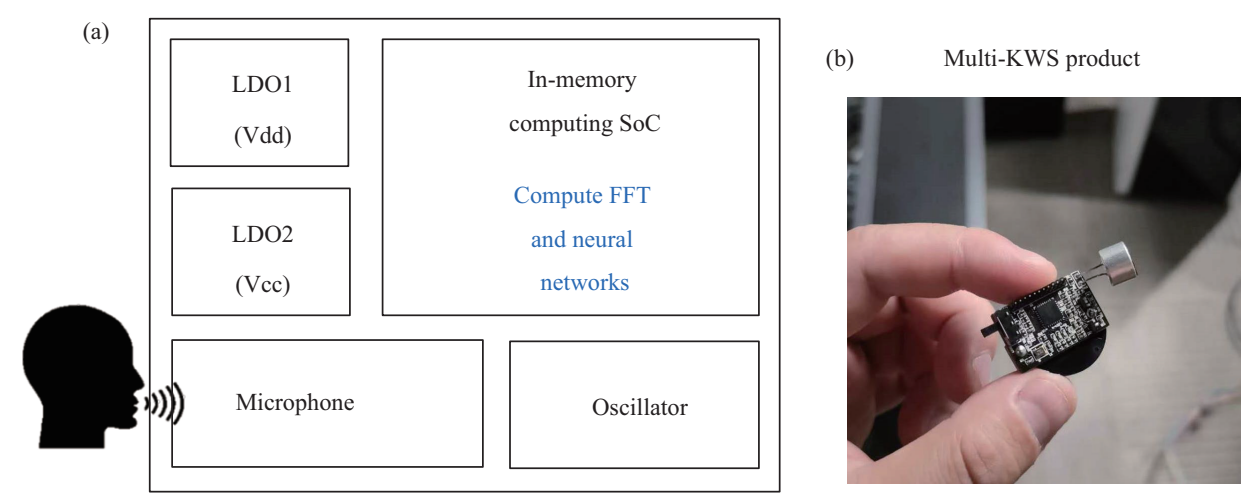

(c)

Performance comparison of various hardware platforms benchmarked by multi-keyword spotting application

\begin{tabular}{|c|c|c|c|c|c|}
\hline $\begin{array}{c}\text { Hardware } \\
\text { platform }\end{array}$ & $\begin{array}{l}\text { Total power } \\
\text { (W) }\end{array}$ & $\begin{array}{l}\text { Dynamic } \\
\text { power }(\mathrm{W})\end{array}$ & $\begin{array}{c}\text { Inference per } \\
\text { second }\end{array}$ & $\begin{array}{l}\text { Energy per } \\
\text { Inference }(J)\end{array}$ & Cost (\$) \\
\hline GPU & 37.83 & 22.86 & 770.39 & 0.0298 & $\$ 250$ \\
\hline $\mathrm{CPU}$ & 28.48 & 11.47 & 1813.6 & 0.0063 & $\$ 200$ \\
\hline JETSON & 4.98 & 2.34 & 419 & 0.0056 & $\$ 300$ \\
\hline MOVIDIUS & 0.647 & 0.437 & 300 & 0.0015 & $\$ 99$ \\
\hline LOIHI & 0.110 & 0.081 & 296 & 0.00027 & N/A \\
\hline Hexagonal & 0.03 & 0.015 & 101 & 0.00015 & $\$ 1$ \\
\hline
\end{tabular}

图 8 (网络版彩图) (a) 完整的语音关键词识别系统的架构, 包括了存算一体化 SoC 芯片、电源 (LDO)、麦克风 和晶振; (b) 语音关键词识别产品的成品照片; (c) 各类硬件平台在执行语音关键词识别任务时的性能比较 ${ }^{[19]}$, 其中 Hexagonal 为本文所实现的硬件平台

Figure 8 (Color online) (a) Architecture of the fully integrated voice-recognition system (including SoC, microphone, and power supplies); (b) a photo of the final product for KWS applications; (c) performance benchmarks of the flash-based in-memory computing SoC versus some existing solutions for multi-keyword spotting applications ${ }^{[19]}$

别系统可以用作实时的多关键词识别引擎, 并表现出高于 $10 \mathrm{TOPS} / \mathrm{W}$ 的能效和接近 $95 \%$ 的推理计 算准确率.

\section{参考文献}

1 Ielmini D, Wong H S P. In-memory computing with resistive switching devices. Nat Electron, 2018, 1: 333-343

2 Yu S. Neuro-inspired computing with emerging nonvolatile memory. Proc IEEE, 2018, 106: 260-285

3 Lee A, Chang M F, Lin C C, et al. RRAM-based 7T1R nonvolatile SRAM with 2x reduction in store energy and 94x reduction in restore energy for frequent-off instant-on applications. In: Proceedings of Symposium on VLSI Technology (VLSI Technology), 2015

4 Aritome S, Shirota R, Hemink G, et al. Reliability issues of flash memory cells. Proc IEEE, 1993, 81: 776-788

5 Lai S K. Flash memories: successes and challenges. IBM J Res Dev, 2008, 52: 529-535

6 Gao S F, Yang G J, Qiu X, et al. Programmable linear RAM: a new flash memory based memristor for artificial synapses and its application to speech recognition system. In: Proceedings of IEEE International Electron Devices Meeting (IEDM), 2019

7 Burr G W, Shelby R M, Sidler S, et al. Experimental demonstration and tolerancing of a large-scale neural network (165 000 synapses) using phase-change memory as the synaptic weight element. IEEE Trans Electron Devices, 2015, 62: $3498-3507$

8 Kim T, Kim H, Kim J, et al. Input voltage mapping optimized for resistive memory-based deep neural network 
hardware. IEEE Electron Device Lett, 2017, 38: 1228-1231

9 Lee S, Jung G, Fouda M E, et al. Learning to predict IR drop with effective training for ReRAM-based neural network hardware. In: Proceedings of the 57th ACM/IEEE Design Automation Conference (DAC), 2020

10 Roy K, Chakraborty I, Ali M, et al. In-memory computing in emerging memory technologies for machine learning: an overview. In: Proceedings of the 57th ACM/IEEE Design Automation Conference (DAC), 2020

11 Chen A. A comprehensive crossbar array model with solutions for line resistance and nonlinear device characteristics. IEEE Trans Electron Devices, 2013, 60: 1318-1326

12 Guo Y H, Shi H H, Kumar H, et al. Spottune: transfer learning through adaptive fine-tuning. In: Proceedings of IEEE/CVF Conference on Computer Vision and Pattern Recognition (CVPR), 2019. 4805-4814

13 He K M, Zhang X Y, Ren S Q, et al. Deep residual learning for image recognition. In: Proceedings of IEEE Conference on Computer Vision and Pattern Recognition (CVPR), 2016. 770-778

14 Cheng M, Xia L X, Zhu Z H, et al. Time: a training-in-memory architecture for memristor-based deep neural networks. In: Proceedings of the 54th Annual Design Automation Conference, 2017

15 Li C, Belkin D, Li Y N, et al. Efficient and self-adaptive in-situ learning in multilayer memristor neural networks. Nat Commun, 2018, 9: 2385

16 Kunze J, Kirsch L, Kurenkov I, et al. Transfer learning for speech recognition on a budget. In: Proceedings of the 2nd Workshop on Representation Learning for NLP, 2017. 168-177

17 Zoph B, Yuret D, May J, et al. Transfer learning for low-resource neural machine translation. In: Proceedings of Conference on Empirical Methods in Natural Language Processing, 2016. 1568-1575

18 Hochreiter S. The vanishing gradient problem during learning recurrent neural nets and problem solutions. Int J Unc Fuzz Knowl Based Syst, 1998, 06: 107-116

19 Blouw P, Choo X, Hunsberger E, et al. Benchmarking keyword spotting efficiency on neuromorphic hardware. In: Proceedings of the 7th Annual Neuro-inspired Computational Elements Workshop, 2019

\title{
Programmable linear random-access memory and its in-memory computing circuits and systems based on flash memory
}

Liang ZHAO ${ }^{1,3}$, Shifan $\mathrm{GAO}^{1}$, Shengbo ZHANG ${ }^{2}$, Xiang $\mathrm{QIU}^{2}$, Fan YANG ${ }^{1}, \mathrm{Jie}^{2}{ }^{3}$, Zezhi $\mathrm{CHEN}^{3}$ \& Yi ZHAO ${ }^{*}$

1. College of Information Science and Electronic Engineering, Zhejiang University, Hangzhou 310027, China;

2. Flash Billion Semiconductor Co. Ltd., Shanghai 201210, China;

3. Hefei Reliance Memory Ltd., Hefei 230088, China

* Corresponding author. E-mail: yizhao@zju.edu.cn

\begin{abstract}
The neural network inference chips based on novel non-volatile memories and compute-in-memory architectures have exhibited significant advantages in terms of power consumption, computation speed and storage density, making them a widely-attractive candidate for IoT and edge computing applications. In this paper, we introduced the design and implementation of an in-memory computing SoC chip based on modified floating-gate transistor devices named PLRAM. In order to achieve highly-efficient inference computation under constrained resources, this system combines a series of techniques from the device, circuit and system perspectives, including: new devices with 7 bit/cell storage capability, self-adaptive write of data, compensation of circuits mismatch and model deployment techniques based on training residual models. These new techniques helped the system to achieve superb overall performance, including compact form factor, energy efficiency above 10 TOPS/W and computation accuracy of roughly $95 \%$. It has been successfully used in low-power, low-cost products for keywordspotting applications.
\end{abstract}

Keywords in-memory computing, system-on-chip, modified floating gate transistor, PLRAM, model deployment techniques 